\title{
Accuracy Improvement of PLIC-VOF Volume-Tracking Method Using the Equation of Surface Normal Vector
}

\author{
Mingyu Sun \\ Institute of Fluid Science, Tohoku University, Sendai, Japan \\ Email: sun.tohoku@gmail.com
}

Received November 13, 2012; revised December 14, 2012; accepted December 21, 2012

\begin{abstract}
The PLIC/SN method that combines the second-order volume tracking method (PLIC-VOF) with the equation of surface normal (SN) vector was recently proposed (M. Sun, "Volume Tracking of Subgrid Particles," International Journal for Numerical Methods in Fluids, Vol. 66, No. 12, 2011, pp. 1530-1554). The method is able to track the motion of a subgrid particle, but the accuracy is not as good as expected on high resolution grids for vortical flows. In this paper, a simple unsplit multidimensional advection algorithm is coupled with the equation of SN vector. The advection algorithm is formulated as the finite volume method, so that it can be used readily for both structured and unstructured grids while maintaining the exact mass conservation. The new method improves the accuracy significantly for high resolution grids. In the well-known test of the time-resolved vortex problem of $T=2$, the circular interface is resolved with an accuracy better than ever using the equation of $\mathrm{SN}$ vector.
\end{abstract}

Keywords: VOF; PLIC; PLIC/SN; Surface Normal; Interface; Level Set; Unstructured Grid

\section{Introduction}

The volume-of-fluid (VOF) is one of the most widely used methods for the numerical simulation of interfacial phenomena [1]. In the VOF method, the evolution of an interface is predicted generally by solving the advection equation

$$
\phi_{t}+\boldsymbol{u} \cdot \nabla \phi=0,
$$

where $\boldsymbol{u}$ is the velocity. Function $\phi$ is the volume fraction or color function at the discrete level. It is unity in a cell filled with one phase, and is zero if the cell lies in the other phase. Because the volume fraction is discontinuous across a sharp interface, it is challenging to advect (1). The volume tracking method, or geometric VOF method, that resolves an interface basically within one cell while maintaining the mass conservation, has been developed for over decades [2,3]. In this method, the interface in a cell is not tracked explicitly, but reconstructed and approximated by a simple geometry, such as simple line (piecewise constant) interface calculation (SLIC) [4] and piecewise linear interface calculation (PLIC). A historic review of the piecewise constant and linear reconstructions can be found in [2,5]. In the PLIC, the surface normal vector, $\boldsymbol{n}$, is required to construct the linear interface

$$
\boldsymbol{n} \cdot \boldsymbol{r}+h=0,
$$

where $h$ is the line constant. The normal vector can be inferred from the spatial distribution of $\phi$, symbolically by,

$$
\boldsymbol{n}=\nabla \phi .
$$

A few numerical methods have been proposed for the calculation of the surface normal from the volume fraction directly by the finite-difference method [6], fitting methods [7-10], or height function technique (e.g. [11]). The difficulty that these methods must overcome is originated from the discontinuous property of the volume fraction. In fact, (3) is just a symbolic representation of $\mathrm{SN}$ vector if $\phi$ is interpreted as the discontinuous volume fraction. One may also integrate the same Equation (1) for a smooth level-set function with the hope that its gradient (3) can be straightforwardly evaluated with a better accuracy, as proposed by Sussman and Puckett [12].

Since it is the surface normal (3) instead of the level set function itself that is required for the geometric VOF, one may directly integrate the equation for the $\mathrm{SN}$ vectors,

$$
\boldsymbol{n}_{t}+\nabla(\boldsymbol{u} \cdot \boldsymbol{n})=0,
$$

which can be readily derived by taking the gradient of (1). Although the volume fraction is discontinuous, the SN vector can be a continuous function near an interface, a clear advantage for numerical analysis using the finite difference method. Raessi et al. [13] investigated the 
equation imposed with the unit normal constraint $(|\boldsymbol{n}|=1)$. We proposed the PLIC/SN method that combines the Equation (4) directly with the PLIC reconstruction and DDR advection algorithm [14], and found that the method allows us to track subgrid particles [15]. The capability of the PLIC/SN method was successfully used to study the fragmentation onset of a liquid drop [16]. The PLIC/SN method improves the accuracy at low grid resolution, but experiences a loss of high-order accuracy on high resolution grids in the time-resolved vortical flows.

In this paper, an unsplit multidimensional advection algorithm is developed to replace the DDR used in the previous PLIC/SN method. This paper is organized as follows. The new advection algorithm used is introduced in Section 2.1, and the positivity of phase volume is enforced by a limiting procedure to be shown in Section 2.1.2. The accuracy of the proposed method is evaluated by simulating two typical test cases, and the results are summarized in Section 3.

\section{The PLIC/SN Method}

The PLIC/SN method that couples the PLIC-VOF volume-tracking method with the $\mathrm{SN}$ equation consists of three procedures [15],

1) to reconstruct the piecewise linear interface with a given surface normal in each interface cell such that the interface truncates the cell with a fractional volume equaling the given phase volume in the cell;

2) to advance the phase volumes using an advection algorithm, which preserves the mass while ensuring all phase volumes lie within the bound of $[0, \Omega]$, where $\Omega$ is the cell volume;

3) to advance the SN vectors by solving the SN equation.

Procedures 1) and 2) are nothing but a typical PLICVOF method except that the SN vectors are given by 3). In the previous work, the DDR was used to evaluate volume fluxes in 2). In this work, a simple multidimensional advection algorithm is coupled with the SN equation.

\subsection{Advection of Phase Volumes}

\subsubsection{Volume Update}

The advection Equation (1) is solved by the finite volume method. The volume flux is evaluated at every face in a unsplit manner. Given an incompressible flow field $\boldsymbol{u}$, the advection equation is rewritten as

$$
\left(\phi_{k}\right)_{t}+\nabla \cdot\left(\boldsymbol{u} \phi_{k}\right)=0
$$

where $\phi_{k}$ is the volume fraction of phase $k$. Integrating (5) over cell $i$ of volume $\Omega$ as the finite volume method, one gets

$$
\left(\Omega_{k}\right)_{i}^{n+1}=\left(\Omega_{k}\right)_{i}^{n}-\sum_{j}\left(\phi_{k j} \Omega_{i j}\right),
$$

where $\Omega_{k}$ is the phase volume satisfying $\Omega_{k}=\phi_{k} \Omega$, and $\phi_{k j}$ represents the fraction of phase $k$ of total volume flux

$$
\Omega_{i j}=u_{i j} s_{j} \Delta t .
$$

where $u_{i j}$ is the outward normal velocity at grid face $j$ of length $s_{j}$. In order to maintain the conservation, we have the saturation constraint $\sum_{k} \phi_{k j}=1$.

The volume fraction at the face $\phi_{k j}$, which is calculated geometrically in PLIC-VOF, is generally different from $\phi_{k}$ that is defined in the cell. Figure 1 shows a few possible method to evaluate $\phi_{k j}$. Consider a control volume or a cell of any shape, which is a rectangular in the figures. We want to find the phase fraction at face AB. Suppose the interface CD has been properly reconstructed (e.g. [15] and there referred), and also suppose the total volume flux $\Omega_{i j}$ is also known. The area of donor polygon ABEF must be the same as the total volume flux, but there is a freedom to define its shape.

The naive unsplit method [2] assumes the donor polygon ABEF is a rectangular as shown in Figure 1(a). The volume flux of the dark phase is then geometrically calculated from the area of polygon ACGF. This method is not recommended because face-adjacent polygons may overlap. The DDR method [14] traces the upwind characteristic inside the upstream cell only, BE in Figure 1(b), and retains the other side bounded by the upstream cell. This method has been coupled with the SN equation in our previous study [15], in which the loss of highorder accuracy for high resolution grids is observed for vortical flows. It was believed that the loss of accuracy is due to the DDR advection algorithm. In order to verify this speculation, the multi-dimensional advection method is coupled with the $\mathrm{SN}$ equation. The idea is similar to what investigated in [2]. This method traces upwind characteristics on two sides, $\mathrm{BE}$ and $\mathrm{AF}$, and the volume flux of the dark phase is set to be the area of polygon ACGF as shown in Figure 1(c). If a long material interface aligned with $\mathrm{CD}$ divides the whole computational domain, this method is obviously more accurate.

\subsubsection{Positivity of Phase Volume}

The conservation of phase volumes cannot ensure the positivity of each phase volume. We introduce a centercentered flux limiter function $\Phi_{i} \in[0,1]$ to reduce the outward volume flux, based on the phase with the smaller volume in the cell,

$$
\tilde{\phi}_{s j}=\Phi_{i} \phi_{s j}
$$

for all faces with positive normal velocity $u_{i j}>0$, such that the remaining volume in the cell will not become 


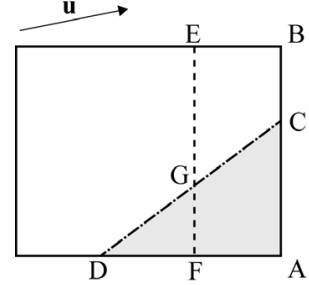

(a)

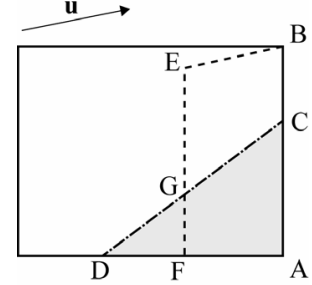

(b)

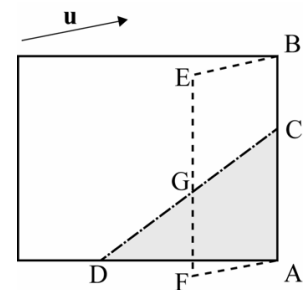

(c)

Figure 1. Calculation of phase volume flux at face AB. Total volume flux are the same for three cases, defined by the area of ABEF. The volume flux of the dark phase is all given by ACGF. (a) Naive unsplit advection method; (b) The DDR method; (c) Multi-dimensional advection method.

negative. The volume fraction of the other phase at the face must be updated to $1-\tilde{\phi}_{s j}$ due to conservation.

A simple and efficient iterative method is used to calculate $\Phi_{i}$. We start from the outward flux of the small phase,

$$
f_{s}^{(0)}=\sum_{j, u_{i j}>0} \phi_{s j} \Omega_{i j},
$$

and then the first estimate of the limiter function can be obtained by,

$$
\Phi_{i}^{(0)}=\left\{\begin{array}{l}
\frac{\Omega_{s}^{n}}{f_{s}}, \text { for } f_{s}>\Omega_{s}^{n} \\
1, \quad \text { otherwise }
\end{array}\right.
$$

where $\Omega_{s}^{n}$ is the volume of the small phase in the last step. It can be easy shown that the small volume remains non-negative if the phase volume is updated by using limited flux (7). This limiter function was directly used in [15].

In the initial estimate, only the outward volume fluxes are considered, which leads to the strictest limiter function. The possible inward volume fluxes that increase the volume are not considered. The limiter function may be further relaxed by an iterative method. With the additional inward fluxes, (8) becomes,

$$
f_{s}^{(m)}=\sum_{j, u_{i j}>0} \phi_{s j} \Omega_{i j}+\sum_{j, u_{i j}<0} \min \left(\phi_{s j}, \tilde{\phi}_{s j}^{(m-1)}\right) \Omega_{i j},
$$

and then $\Phi_{i}^{(m)}$ can be obtained similarly by (9). Superscript $m$ denotes the number of iterations. Notice that the limiter function (7) tends to reduce the outward flux of the small volume, and to increase that of the other large volume due to conservation. Since the small phase in two cell may be different, the inward flux is calculated by the minimum possible flux, $\min \left(\phi_{s j}, \tilde{\phi}_{s j}^{(m-1)}\right)$. Figure 2 records the geometrical errors in simulating a circular interface in a time-resolved vortical velocity field, and the details of this test will be introduced in Section 3. The geometric error is reduced dramatically with the first iteration; more iterations hardly affect the overall accuracy. Fixed three iterations are performed for all other tests in this paper.

It emphasized that the limiting procedure is general for any advection algorithm, and is valid for any structured and unstructured grid system, given the volume flux of the small phase and the total volume flux at faces. There is no need to deal with complicated geometrical problems, such as polygon volume overlapping and/or underlapping. The limiting procedure maintains the exact volume conservation and the boundedness of phase volumes, if the total volume flux at cell faces is defined from a divergence-free velocity field.

\subsection{Discretization of Surface Normal Equations}

In numerical simulation, Equation (4) are reformulated in a non-conservative form [15], using $l_{y}=m_{x}$,

$$
\boldsymbol{n}_{t}+\left(\boldsymbol{F}_{1}\right)_{x}+\left(\boldsymbol{F}_{2}\right)_{y}=\boldsymbol{S}
$$

where

$$
\boldsymbol{F}_{1}=\left(\begin{array}{c}
u l \\
u m
\end{array}\right), \boldsymbol{F}_{2}=\left(\begin{array}{c}
v l \\
v m
\end{array}\right), \boldsymbol{S}=\left(\begin{array}{c}
-m v_{x}+l v_{y} \\
m u_{x}-l u_{y}
\end{array}\right),
$$

and $(l, m),(u, v)$ are two components of SN vector $\boldsymbol{n}$ and velocity $\boldsymbol{u}$ respectively. We use the two-step RungeKutta method for achieving second-order accuracy in time, and the MUSCL method for the accuracy in space. The details of the numerical method have been reported in [15].

\section{Numerical Results and Discussion}

In all test cases, the computational domain is a square of

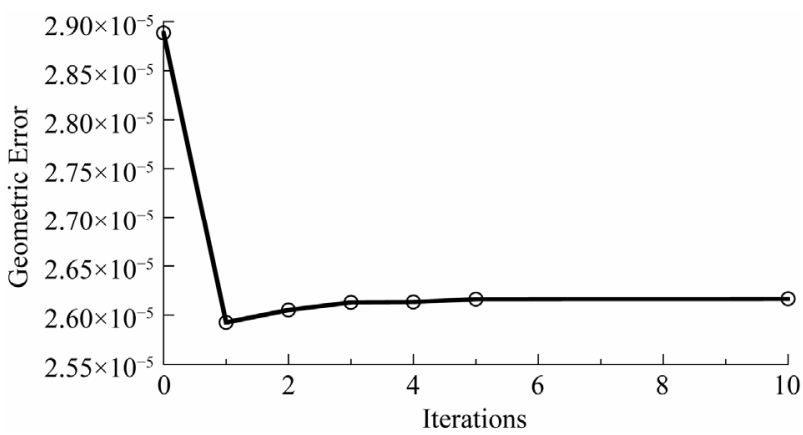

Figure 2. Effect of the limiter function on the geometric error. 
$(0,1)^{2}$, with open or free boundary outside. Initial surface normal vectors are specified the same as [15]. The initial phase volume intersected with the interface is exactly calculated; the sum of all phase volumes inside a circle differs from the exact area by the order of $O\left(10^{-16}\right)$. The velocities at cell faces are also exactly specified, to exclude any possible error in the treatment of velocity. The CFL number is always taken as $1 / 8$, the same as [13]. The geometric error measure, $L_{1}$ norm, is defined as

$$
\epsilon_{g}=\sum_{j=1}^{N} \Omega_{j}\left|\phi_{\text {cal }}-\phi_{\text {exact }}\right|_{j}
$$

where $\phi_{\text {cal }}$ and $\phi_{\text {exact }}$ are the calculated and the exact volume fractions in cell $j$ with volume $\Omega_{j} . N$ is the total number of cells used in the domain. The error measure for surface normal vector is defined as

$$
\epsilon_{n}=\frac{1}{N_{I}} \sum_{j=1}^{N_{I}}\left(\left|\tilde{l}_{\text {cal }}-\tilde{l}_{\text {exact }}\right|_{j}+\left|\tilde{m}_{\text {cal }}-\tilde{m}_{\text {exact }}\right|_{j}\right),
$$

where the tilde indicates the surface normal has been normalized. $N_{I}$ is the total number of interface cells; only the error in these interface cells is considered. Both the numerical and exact surface normal vectors are defined at the cell center.

\subsection{Translation of Circular Surfaces}

The first test is the translation of a circular interface. The initial circle of 0.3 in diameter centered at $(0.25,0.5)$ is translated in the constant velocity field of $(u, v)=(1,0)$ for $t=0.5$. The test was taken from $[13,15]$. The geometric errors for this test are presented in Table 1. It is apparent that the grid convergence has been achieved by the present PLIC/SN method, even for circles as small as $1.2 \Delta x$. In this test, the flow velocity is perpendicular to the vertical faces; the DDR advection is actually the same as the present multidimensional one. The geometrical errors obtained are actually identical. These errors are solely due to the advection algorithms, since the sur-

Table 1. Geometrical errors in translating a circle $(u=1, v$ $=\mathbf{0})$.

\begin{tabular}{ccccc}
\hline Mesh & \multicolumn{4}{c}{ Geometrical errors } \\
\hline$\Delta x$ & $\mathrm{~d} / \Delta x$ & Raessi [13] & DDR [15] & Present \\
\hline $1 / 4$ & 1.2 & - & $2.0 \times 10^{-2}$ & $2.00 \times 10^{-2}$ \\
$1 / 8$ & 2.4 & - & $3.9 \times 10^{-3}$ & $3.99 \times 10^{-3}$ \\
$1 / 16$ & 4.8 & - & $1.1 \times 10^{-3}$ & $1.09 \times 10^{-3}$ \\
$1 / 32$ & 9.6 & $4.1 \times 10^{-4}$ & $4.5 \times 10^{-4}$ & $4.46 \times 10^{-4}$ \\
$1 / 64$ & 19.2 & $1.1 \times 10^{-4}$ & $1.1 \times 10^{-4}$ & $1.06 \times 10^{-4}$ \\
$1 / 128$ & 38.4 & $2.0 \times 10^{-5}$ & $1.9 \times 10^{-5}$ & $1.93 \times 10^{-5}$ \\
\hline
\end{tabular}

face normal vectors of a linear spatial distribution are all exactly solved using the second-order finite different scheme for this simple velocity field. The volume differences between the initial area of the circle and the final area for all grids are the order of $10^{-16}$; the mass conservation is maintained within the round-off error.

\subsection{A Circular Fluid Body in Time-Resolved Vortex Field}

The second test is the deformation of a circular surface of $d=0.3$ in a vortex velocity field. The circle is initially centered at $(0.5,0.75)$. The time-resolved velocity field is specified as,

$$
\begin{gathered}
u=-\cos (\pi t / T) \sin ^{2}(\pi x) \sin (2 \pi y), \\
v=\cos (\pi t / T) \sin ^{2}(\pi y) \sin (2 \pi x) .
\end{gathered}
$$

The test, taken from [2], is a standard case for evaluating the accuracy of sharp interface methods. The vortical velocity field will deform the circle and promote topology changes. It is periodic with a period of $T$. The circle will undergo the maximum deformation at $t=T / 2$, and then return to its initial state. Error measurements are performed on the differences in data observed between $t=0$ and $t=T$.

Results of the circle after one period are shown in Figure 3 for the period $T$ varying from 0.5 to 4.0 . The exact solution is a perfect circle as the initial state. Linear segments reconstructed in all interface cells with $\phi \in(0,1)$ are plotted. They are not contours of equal volume fractions. Surface normal vectors in the interface cells and the cells filled with the dark phase are also plotted. The vectors in other cells are used in computation as well, but not plotted for the sake of clarity. The arrow indicates the direction of the normal vector, starting from the cell center, and its length is proportional to the magnitude of the vector.

It is seen that for small period $T \leq 2$, the circle is resolved excellently well even on the $32^{2}$ grid. The surface normal errors increase dramatically for large period $T=4$. The location where the SN vector is of zero magnitude should correspond to the centroid of the circle to the second-order accuracy [15]. However, the SN vector of zero magnitude deviates far from the center $(0.5,0.75)$ in Figure 3(d), compared with those in other three figures of smaller periods.

The dependence of numerical error on the period is further investigated. Both the geometric and the SN vector errors are plotted in Figure 4(a). The present method is more accurate than that obtained by Rider \& Kothe [2] for $T \leq 2$. However, the geometric error increases rapidly for large period. As seen from Figure 4(a), the SN error increases nearly four orders of magnitude for the period $T$ increasing from 0.5 to 8.0 . The second-order 


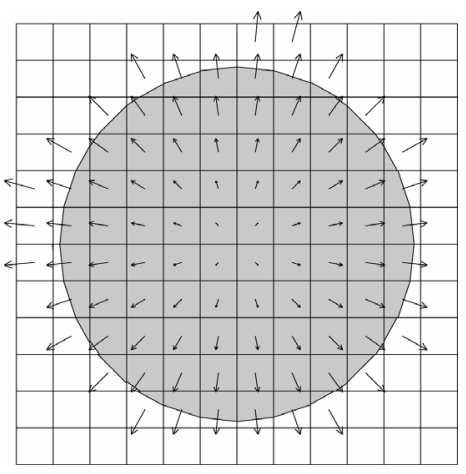

(a)

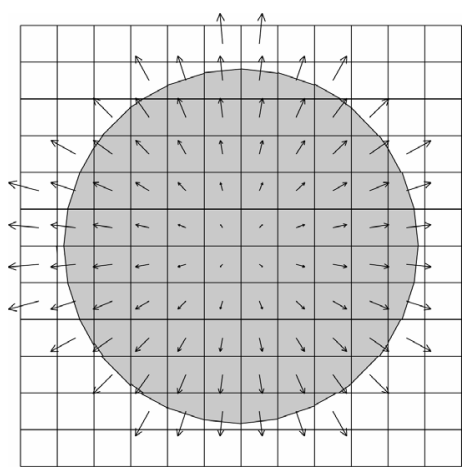

(b)

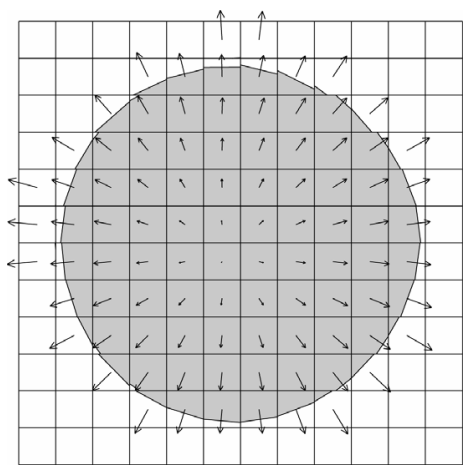

(c)

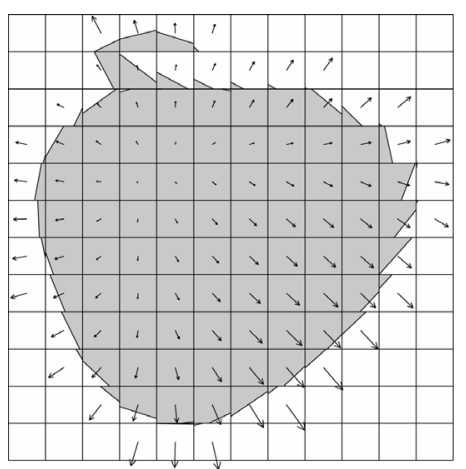

(d)

Figure 3. Results for the circular fluid body placed in the time-reversed single-vortex flow field on a $32^{2}$ grid, after one peirod $T$ : (a) $T=0.5$; (b) $T=1.0$; (c) $T=2.0$; (d) $T=4.0$. The square domain is 0.375 wide and high, centered at $(0.5$, 0.75). accuracy is generally achieved for all periods as shown in Figure 4(b). It is of interest that the geometric errors show a second-order convergence rate even for $T=8$.

The error increase for large period is closely related to the difficulty in integrating the SN equation for large period, in which the interface structures experiences a strong deformation. The circle undergoes the maximum deformation at $t=T / 2$. The grid dependence of numerical solution of $l$, the $x$-component of $\mathrm{SN}$ vector, are plotted in Figure 5 for $T=2.0$ and $T=8.0$. At this moment, the interface is nearly circular, so the magnitude of the $y$-component of $\mathrm{SN}$ vector is much smaller, and will not be discussed here. For the small period of $T=2$ shown in Figure 5(a), a $32^{2}$ grid can resolve the SN vector well in central domain where the deformed circle is located. However, for the period of $T=8$, the wavy

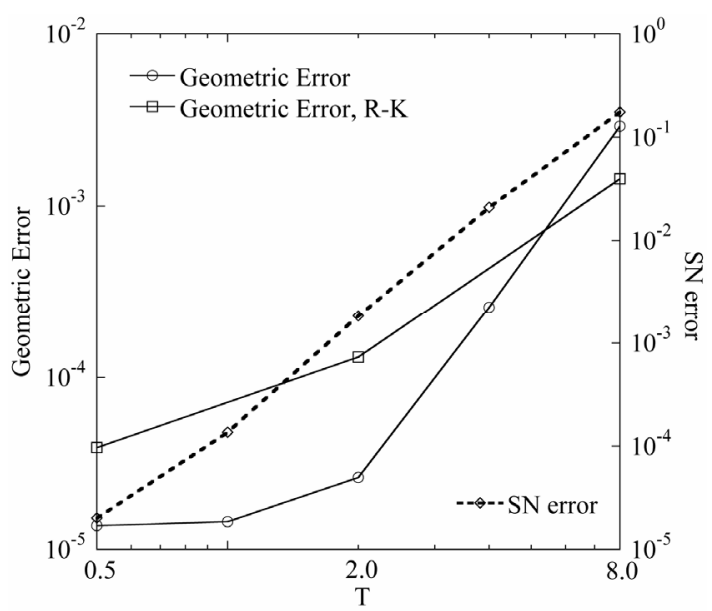

(a)

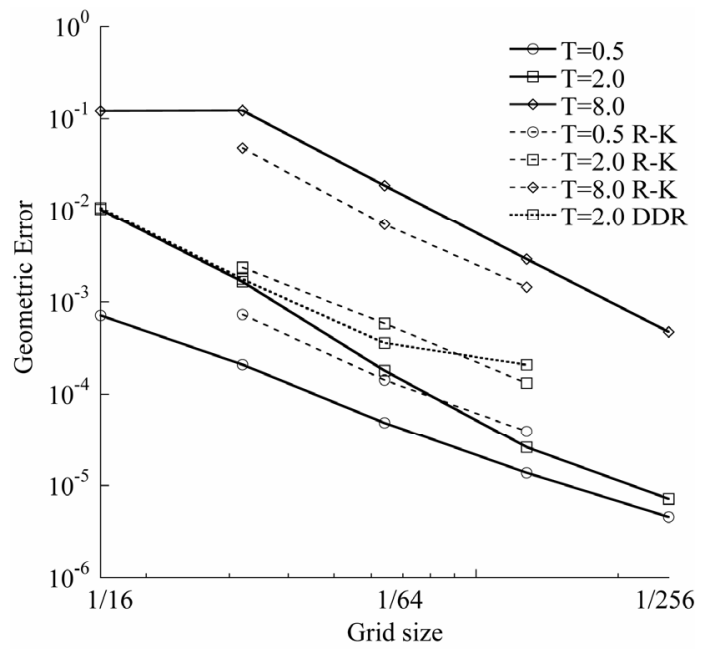

(b)

Figure 4. Results for the circular fluid body placed in the time-reversed single-vortex flow field: (a) Geometric and SN vector errors on a $128^{2}$ grid for $T=0.5,1.0,2.0,4.0$ and 8.0; (b) Geometric errors against grid size. Results are compared with those of Rider \& Kothe [2] and DDR [15]. 
Table 2. Convergence of the present method that couples the multidimensional advection algorithm with the SN vector equation in the reversed single-vortex test. A few published results obtained using various advection and reconstruction methods are compared.

\begin{tabular}{cccccccc}
\hline Mesh & \multicolumn{2}{c}{ SN vector } & \multicolumn{2}{c}{ Youngs } & \multicolumn{2}{c}{ Puckett } & Quadratic \\
\hline$\Delta x$ & Present & DDR [15] & H \& F [17] & EMPA [9] & R \& K [2] & EMPA [9] & EL-LE [8] \\
\hline $1 / 8$ & $4.70 \times 10^{-2}$ & $4.74 \times 10^{-2}$ & - & - & - & - & - \\
$1 / 16$ & $1.02 \times 10^{-2}$ & $1.06 \times 10^{-2}$ & - & - & - & - & - \\
$1 / 32$ & $1.65 \times 10^{-3}$ & $1.76 \times 10^{-3}$ & $2.49 \times 10^{-3}$ & $2.29 \times 10^{-3}$ & $2.36 \times 10^{-3}$ & $2.14 \times 10^{-3}$ & $1.88 \times 10^{-3}$ \\
$1 / 64$ & $1.80 \times 10^{-4}$ & $3.58 \times 10^{-4}$ & $7.06 \times 10^{-4}$ & $6.72 \times 10^{-4}$ & $5.85 \times 10^{-4}$ & $5.39 \times 10^{-4}$ & $4.42 \times 10^{-4}$ \\
$1 / 128$ & $2.61 \times 10^{-3}$ & $2.08 \times 10^{-4}$ & $2.23 \times 10^{-4}$ & $2.24 \times 10^{-4}$ & $1.31 \times 10^{-4}$ & $1.29 \times 10^{-4}$ & $9.36 \times 10^{-5}$ \\
\hline
\end{tabular}

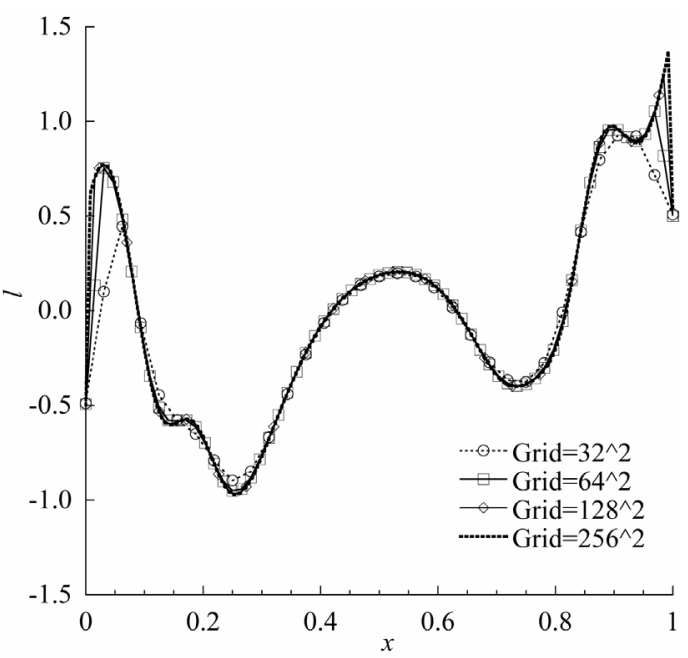

(a)

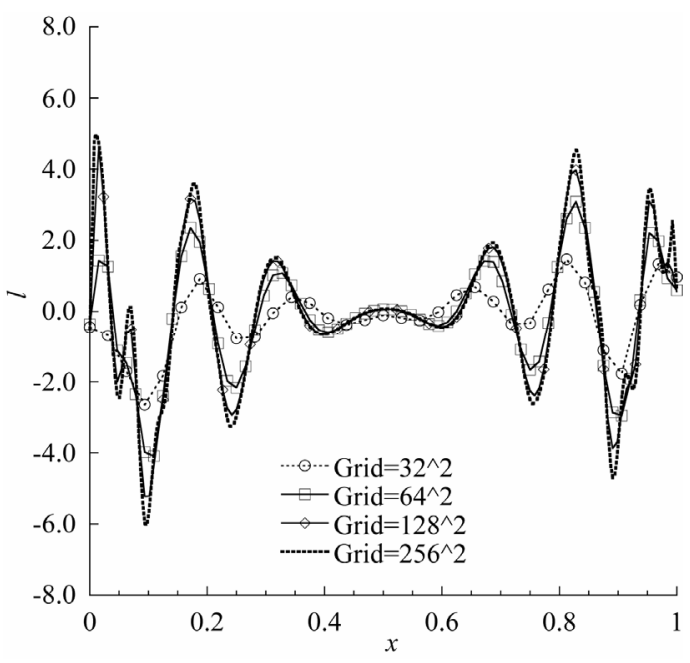

(b)

Figure 5. Numerical solution of $x$-component of SN vector (I) along $y=0.5$ at $t=T / 2$ : (a) $T=2$; (b) $T=8$.

structure of the SN vector imposes a great difficulty for the solution to converge. The numerical error of the SN vector introduced in the stage of large deformation may remain and affect the solution at last moment.

The geometric errors of the PLIC/SN method are compared with other results in literature in Table 2 . The present results show an improved accuracy for all grids. The grid convergence is achieved for grid resolutions as low as $\Delta x=1 / 8$. Compared with the DDR advection, the geometric errors at high resolution grids $(\Delta x=1 / 64,1 / 128)$ have been greatly reduced. For $\Delta x=1 / 128$, the geometrical error $\left(2.61 \times 10^{-5}\right)$ is only a bit larger than that in the translation test. To what we know, this accuracy has never been reported before.

\section{Concluding Remarks}

It has been shown shown that the equation of allow us to implicitly track a subgrid particle [15] when coupled with the PLIC-VOF method. This work confirms that the equation of $\mathrm{SN}$ vector coupled with the multidimensional advection algorithm improves the accuracy not only on low resolution grids but also on high resolution grids in the vortical flows with a low period. The geometric error obtained is much lower than those reported in literature.

The PLIC/SN method generally improves the accuracy in the calculation of SN vector, especially at low grid resolution. It can resolve a particle with less cells, or resolve more particles/bubbles with a given grid. In the direct simulation of interfacial phenomena with a large amount of particles/bubbles, such as defragmentation, cavitation, it is expected that the PLIC/SN can resolve interfacial structure with one order of magnitude less grid cells for achieving the same interface resolution.

\section{REFERENCES}

[1] C. W. Hirt and B. D. Nichols, "Volume of Fluid (VOF) Method for the Dynamics of Free Boundaries," Journal of Computational Physics, Vol. 39, No. 1, 1981, pp. 201225. doi:10.1016/0021-9991(81)90145-5

[2] W. J. Rider and D. B. Kothe, "Reconstructing Volume Tracking," Journal of Computational Physics, Vol. 141, No. 2, 1998, pp. 112-152. doi:10.1006/jcph.1998.5906 
[3] R. Scardovelli and S. Zaleski, "Direct Numerical Simulation of Free-Surface and Interfacial Flow," Annual Review of Fluid Mechanics, Vol. 31, 1999, pp. 567-603. doi:10.1146/annurev.fluid.31.1.567

[4] W. F. Noh and P. Woodward, "SLIC (Simple Line Interface Calculation)," In: A. I. van der Vooren and P. J. Zandbergen, Eds., Lecture Notes in Physics, Springer, New York, 1976, p. 330.

[5] M. Rudman, "Volume-Tracking Methods for Interfacial Flow Calculations," International Journal for Numerical Methods in Fluids, Vol. 24, No. 7, 1997, pp. 671-691. doi:10.1002/(SICI)1097-0363(19970415)24:7<671::AIDFLD508>3.0.CO;2-9

[6] D. L. Youngs, "An Interface Tracking Method for a 3D Eulerian Hydrodynamics Code," Technical Report 44/92/ 35, AWRE, 1984.

[7] E. G. Puckett, “A Volume of Fluid Interface Tracking Algorithm with Applications to Computing Shock Wave Rarefraction," Proceedings of the 4th International Symposium on Computational Fluid Dynamics, 1991.

[8] R. Scardovelli and S. Zaleski, "Interface Reconstruction with Least-Square Fit and Split Eulerian-Lagrangian Advection," International Journal for Numerical Methods in Fluids, Vol. 41, No. 3, 2003, pp. 251-274. doi:10.1002/fld.431

[9] J. López, J. Hernández, P. Gómez and F. Faura, "A Volume of Fluid Method Based on Multidimensional Advection and Spline Interface Reconstruction," Journal of Computational Physics, Vol. 195, No. 2, 2004, pp. 718742. doi:10.1016/j.jcp.2003.10.030

[10] J. E. Pilliod Jr. and E. G. Puckett, "Second-Order Accurate Volume-of-Fluid Algorithms for Tracking Material Interfaces," Journal of Computational Physics, Vol. 199,
No. 2, 2004, pp. 465-502. doi:10.1016/j.jcp.2003.12.023

[11] M. M. Francois and B. K. Swartz, "Interface Curvature via Volume Fractions, Heights, and Mean Values on Nonuniform Rectangular Grids," Journal of Computational Physics, Vol. 229, No. 3, 2010, pp. 527-540. doi:10.1016/j.jcp.2009.10.022

[12] M. Sussman and E. G. Puckett, "A Coupled Level Set and Volume-of-Fluid Method for Computing 3D and Axisymmetric Incompressible Two-Phase Flows," Journal of Computational Physics, Vol. 162, No. 2, 2000, pp. 301337. doi:10.1006/jcph.2000.6537

[13] M. Raessi, J. Mostaghimi and M. Bussmann, "Advecting Normal Vectors: A New Method for Calculating Interface Normals and Curvatures When Modeling Two-Phase Flows," Journal of Computational Physics, Vol. 226, No. 1, 2007, pp. 774-794. doi:10.1016/j.jcp.2007.04.023

[14] D. J. E. Harvie and D. F. Fletcher, "A New Volume of Fluid Advection Algorithm: The Defined Donating Region Scheme," International Journal for Numerical Methods in Fluids, Vol. 35, No. 2, 2001, pp. 151-172. doi:10.1002/1097-0363(20010130)35:2<151::AID-FLD8 7>3.0.CO;2-4

[15] M. Sun, "Volume Tracking of Subgrid Particles," International Journal for Numerical Methods in Fluids, Vol. 66, No. 12, 2011, pp. 1530-1554. doi:10.1002/fld.2331

[16] D. Igra and M. Sun, "Shock-Water Column Interaction, from Initial Impact to Fragmentation Onset," AIAA Journal, Vol. 48, No. 12, 2010, pp. 2763-2771. doi:10.2514/1.44901

[17] D. J. E. Harvie and D. F. Fletcher, "A New Volume of Fluid Advection Algorithm: The Stream Scheme," Journal of Computational Physics, Vol. 162, No. 1, 2000, pp. 1-32. doi: $10.1006 / \mathrm{jcph} .2000 .6510$ 